\title{
REFLECTIONS ON THE ORIGIN OF LIFE MORE THAN AN 'EVOLUTIONARY' PROBLEM
}

\author{
Kepa Ruiz-Mirazo And Álvaro Moreno
}

This paper argues that the question of the origin of life cannot be explained by appealing exclusively to Darwinian evolutionary mechanisms, as many experts tend to assume, but requires a profound change in perspective. Accordingly, we highlight the fact that, in order to operate as a diversification force (and indirectly, a force for a potential increase in complexity), natural selection requires a number of conditions to be met in order for it to be possible: specifically, self-sustained and self-(re-)productive chemical organisation within a sufficiently large phenotypic space (that is, a wide range of functions). Therefore, we suggest an extension of the "self-organising" paradigm towards a "self-(re-)productive» one as an alternative to the main proposals regarding the origin of life, based on molecular populations (typically RNA) subject to Darwinian evolution. Such a paradigm would adequately portray the specificity of the biological phenomenon (particularly, its metabolic and cellular dimension) and would be relevant before, during, and after natural selection started to operate.

Keywords: self-organisation, protocells, metabolism, phenotypic/functional diversity, open evolution.

Many scientists and philosophers currently share the idea that the origin of living entities starting from inert matter, while still a complicated and unresolved problem, is, we may say, «in the process of being solved». Obviously, there is still a great number of «details» to work out, some of them, surely, difficult issues for experts in the field, yet from a global perspective, it is assumed that we can achieve comprehension of this fundamental transition, thanks to the results of Miller's experiments and their many follow-ups (i.e., to the field of «prebiotic chemistry», which has been able to synthesise several relevant compounds for life) and to the theory of evolution, applied to the first selfreplicating molecules (RNA nucleotides or analogues). However, some critics have called this optimistic view into question. For instance, T. Nagel (2012) suggests in his recent book, Mind and cosmos: Why the materialist neo-Darwinian conception of nature is almost certainly false, that contemporary biological sciences are unable to explain the origin or life or the origin of the mind. More specifically, Nagel claims that no scientific theory can provide a satisfying explanation - which, for him, would be a reductionist explanation of physical science- about the possible origin of such complex phenomena from the evolution of the physicochemical world. In his book Nagel tends to identify the meaning of «reductionism» as the equivalent of «reductive materialism», because he considers «emergentist» theories to lack an alternative explanation for these kinds of highly complex systems or their respective cognitive or biological properties. In his own words: «That such purely physical elements, when combined in a certain way, should necessarily produce a state for the whole that is not constituted out of the properties and relations of the physical parts still seems like magic» (Nagel, 2012, p. 55-56).

Because, indeed, a meticulous consideration of the differences between living and non-living 
matter should make us reflect more on the problems around the issue of the origin of life. Explaining the transition between the physicochemical world and the biological world is an enormous challenge, mainly due to the current sharp contrast between them. Any known living being, even unicellular entities, is orders of magnitude more complex (in composition, diversity of interactions, dynamic behaviour, etc.) than the different molecular systems from which it has been postulated to come. When we analyse the components, the chemical transformations, the control mechanisms underlying the properties of the most simple cells on our planet (prokaryotes with very limited genomes), it is impossible to understand at once everything that is happening within it and its relationship with the environment. Countless (bio)chemical synthesis reactions, along with transport, transduction, recognition and signalling processes, among others, occur every second so that the organism is sustained, adapted and can, potentially, reproduce. This is possible thanks to a battery of molecular mechanisms generated by the living beings (particularly, metabolic mechanisms, autonomous synthesis and regulatory control [RuizMirazo \& Moreno, 2012]), which make them especially robust, despite the fact that they are open systems, far from a thermodynamic equilibrium. No other kind of known material system, natural or artificial, displays complexity levels in structure, organisation, or function that are in any way comparable.

The contrast between them is very obvious when we examine each living being individually, but also when we consider the biological phenomenon from a broader time perspective. In time scales much larger than the physiological, life manifests as evolution, that is, as the historical chain of fleeting entities or organisations, which nonetheless pass on their traits to their successors. It is true that, if we extend the frame enough, everything (even an atom, which abides by physical laws) can be understood as the result of a transformation process, of an evolution. However, once the basic pieces and interaction rules are established, matter has implemented surprisingly different long-term evolution modes. On the one hand, the inanimate matter we know has been governed strictly by the fundamental laws that characterise our expanding physical universe, generating stars, planets, asteroid discs, interstellar dust, galaxies and black holes. On the other hand, matter that has integrated (even if only locally and temporarily) biological systems contributes to the development of much more sophisticated and indirect mechanisms of control over its variation, although they are completely coherent with physicochemical laws.

These molecular variation control mechanisms, which, interestingly, appeared in precarious stability conditions (stationary states far from thermodynamic equilibrium), and were then fixed, more and more consistently, in different populations of protoorganisms, to which they granted greater metabolic robustness and adaptability. Thus they managed to transform, through the process of the origin of life, into increasingly complex and, at the same time, more lasting modules, whose life span was several orders of magnitude longer than the proto-organisms from which they came. Then, some polymers, inclined towards copying and preserving their sequence, turned them into partial, but reliable, «molecular records» (Pattee, 1969) of the structural and organisational complexity of the cells in which they resided, where they grew and gained meaning. It was the emergence and functionality of these «record components», which could replicate and reliably transfer to other similar cell systems, that allowed life to become sustainable on Earth in the long term, thanks to a matter evolution method that had been inaccessible up to that moment: Darwinian (Lewontin, 1970) or open evolution (Ruiz-Mirazo \& Moreno, 2012). As astonishing as it may seem, we currently have very solid evidence that the change and re-invention method that characterises living entities, based on populations of genetically instructed metabolisms, has been working for thousands of millions of years, at least on our planet.

\section{NATURAL SELECTION DOES NOT EXPLAIN THE ORIGIN}

The importance of the biological evolutionary process and its enormous temporal depth, as proven by Darwin - and supported by all the subsequent biology - has supplied these molecular control mechanisms (genetic inheritance machinery) with a 
Molecular and organisational complexity

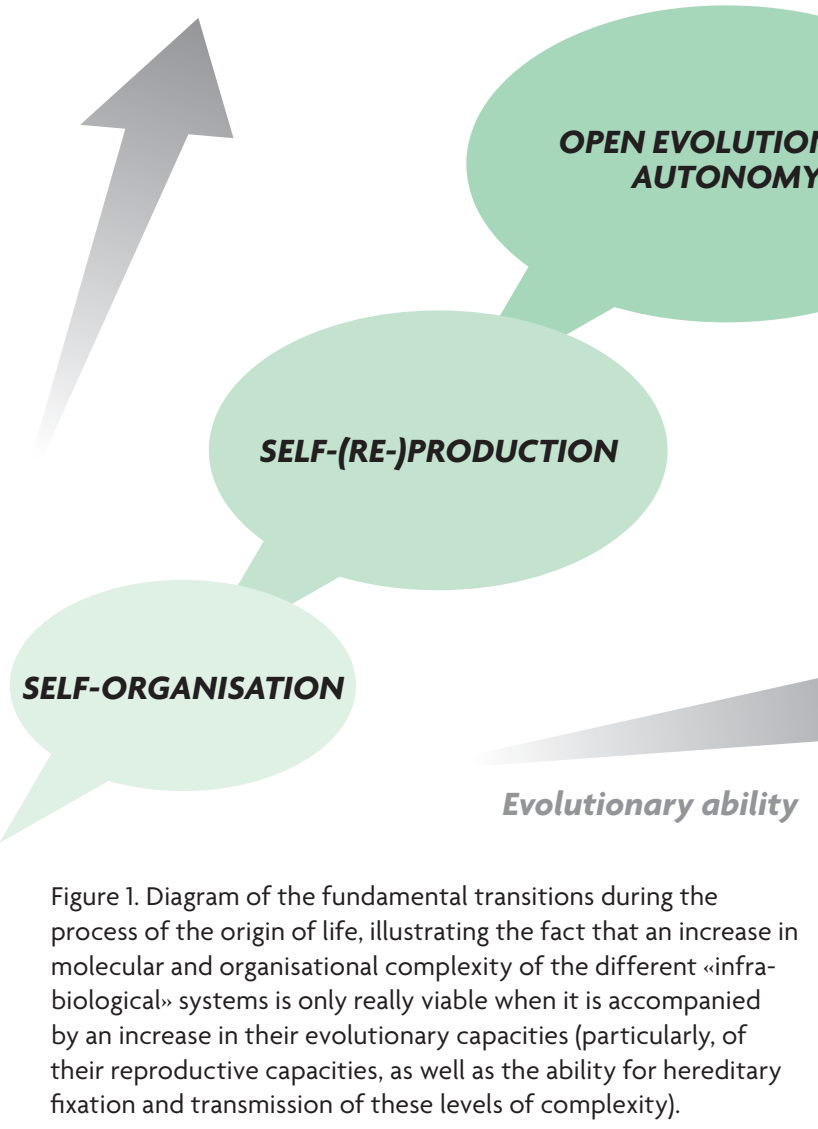

fundamental role in the spread of the reign of the living things on Earth. Together with the lack of an appropriate theory for biological organisation (about which we will talk later), it has generated an important imbalance captured in conceptions which are probably wrong - or, at least, strongly unbalanced - about the problem of the origin of life. We refer, particularly, to the assumption that the theory of evolution (and, more specifically, the mechanism of «natural selection» applied to populations of self-replicating molecules, such as RNAs) is the key to solving the problem of the transition between the domain of the inert and of the living. This assumption, widely accepted in scientific circles for decades (Eigen \& Schuster, 1979; Orgel, 1992; Szostak, 2012), is paradoxically contrary to Darwin's own intuition. Despite having a very advanced view of the origin (similar to the one that appeared a century later), he considered the science of the time, including the theory of evolution that he was promoting, limited when trying to tackle the issue (Peretó, Bada, \& Lazcano, 2009). Actually, what brought experts to consider natural selection as the main -for some, even the only- «driving force» in the process of the emergence of life was a simplistic interpretation of Darwin's legacy. However, as we argued in more detail elsewhere (Moreno \& Ruiz-Mirazo, 2009), relying on authors such as Lewontin (1970) or Wicken (1987) among others, establishing a Darwinian evolutionary mode which actually operates through natural selection, requires a sufficiently broad phenotypic space as a primary condition, that is, a potential for functional variation and diversification that is near impossible to achieve for material organisation methods within a complexity threshold similar to that of proto-cells with their own metabolism. In other words, the natural selection evolutionary mechanism is not useful for explaining the origin of life because, ultimately, natural selection implies the existence of organisms or proto-organisms with a sufficiently rich phenotype (or a set of functions attributable to its components). Then, where can we look for the first stages or mechanisms to help us understand how, under earlyEarth conditions, a sustained change process could start and lead to the emergence of life? Are there any laws or principles in the physical world which allow us to understand the appearance of life as a necessity or should we resign ourselves to think, as Monod (1970) said, that the origin of life is so unlikely, so much the result of sheer chance, that it is practically a miracle? How can matter originate something that seems to be so profoundly different from its initial properties?

\section{WHAT IS THE CHANGE OF PERSPECTIVE THAT WE PROPOSE?}

A good starting point that guarantees full consensus within the scientific community is that nature does not take leaps of faith and, therefore, a continuum must be established between non-living matter and life. In other words, there must be some kind of progressive explanation in increasingly complex 


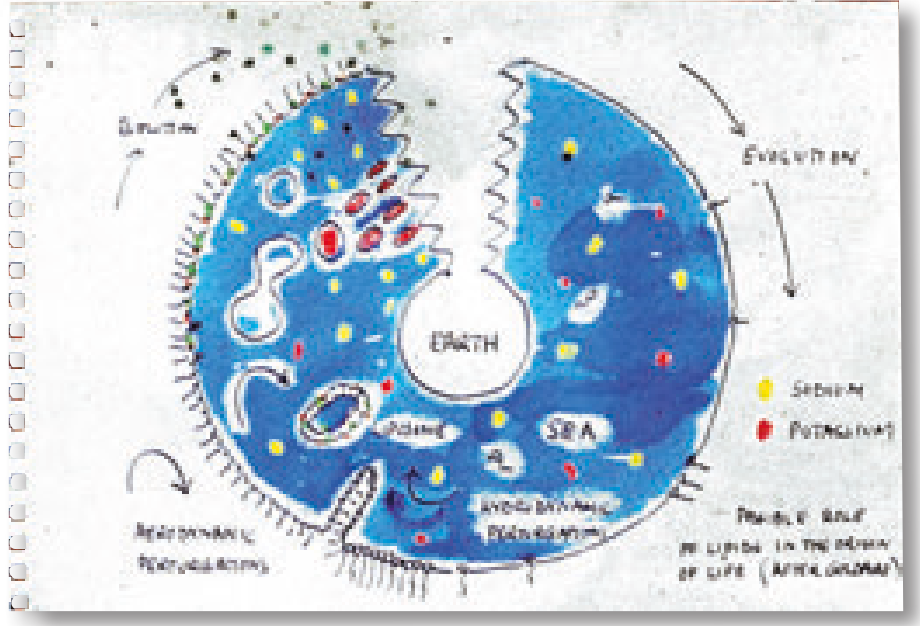

Figure 2. Personal drawing by Alec Bangham (courtesy of David Deamer), in which the author depicts his thoughts on the role of liposomes in the origin of life and the evolutionary capacities that could be developed.

stages (see Figure 1), for the transition to life, although at the limit, we may be bound to a historical narrative, mainly contingent, of the process. As Fry (2000) pointed out, the fundamental problem of the origin of life resides in the tension between the continuity principle and the difficulty of explaining the obvious differences between non-living and living matter. If the origin of life is a legitimate scientific question (and we think it is), we should look for a theory to bridge the gap between physics and biology and experimentally prove that such a bridge (or an important part of it) can be built de novo. In the end, living beings are made of the same materials as non-living systems, so it does not seem unreasonable to clarify the link between one and the other.

From the point of view of physical science, explaining the origin of life is a very difficult task because the more complex a system, the less likely it is to emerge and persist. As a first approximation, it seems quite easy to understand how simple material aggregates can spontaneously generate relatively stable complex structures (complex molecules, macromolecules, or supramolecular aggregates), by virtue of the different kinds and degrees of strength in nature (Simon, 1962): indeed, as a result of these physical interactions with different magnitudes, the formation of more complex structures is to be expected (with more properties that might be considered «emergent», not present in their separate parts - such as, for instance, superconductivity, chemical affinity, or the closed topology of a lipid vesicle). But as structure complexity grows, its sustenance becomes a problem: thermal noise increases fragility and, on the other hand, the coincidence or coordination of many highly specific processes becomes more and more unlikely. Furthermore, the complexity of life resides not only in the structure of its components, but in an intricate network of dynamic interactions, established in conditions far from the thermodynamic equilibrium point. In other words, we are forced to face the problem in organisational terms. Biological systems, in fact, use the self-organising properties of matter in very different ways (Karsenti, 2008), some further away from the equilibrium point than others. Thanks to the advances in the supramolecular and thermodynamic chemistry of irreversible processes during the second half of the twentieth century, we have an adequate framework to understand the generation of relatively complex dynamic organisations, which could even combine different supramolecular aggregates and «dissipative system» order patterns.

However, life goes beyond that: it does not only organise pre-existing material, but also continually generates and regenerates most of it and, at the same time, controls most of the conditions in which such a dynamic organisation occurs. That is, the whole biological system constructs a dynamic organisation through the synthesis and transformation of its components, including those that ensure their own viability and sustenance over time. There are many implications of the change from self-organisation to self-production, but they can be summarised in two points (Ruiz-Mirazo \& Moreno, 2012): (i) a much more important internal cohesion and dynamic robustness, based on the «functional integration» of the system components; and (ii) an emerging control over the environmental conditions under which the system operates: that is, the appearance of «agency». All of this is related, in short, with the emergence of metabolic systems: this problem reveals the fact that if we aspire to a solid and coherent general theory about biological organisation, it must surely take

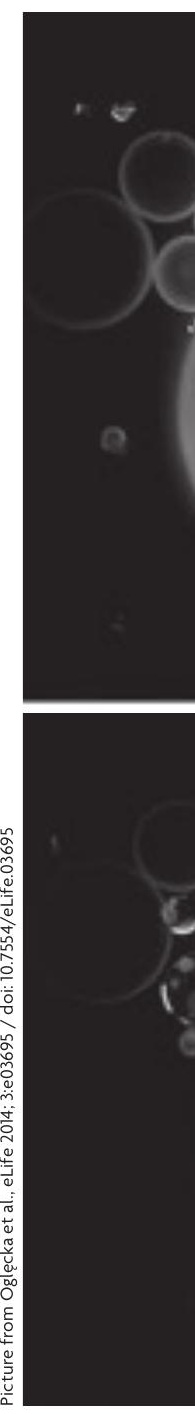



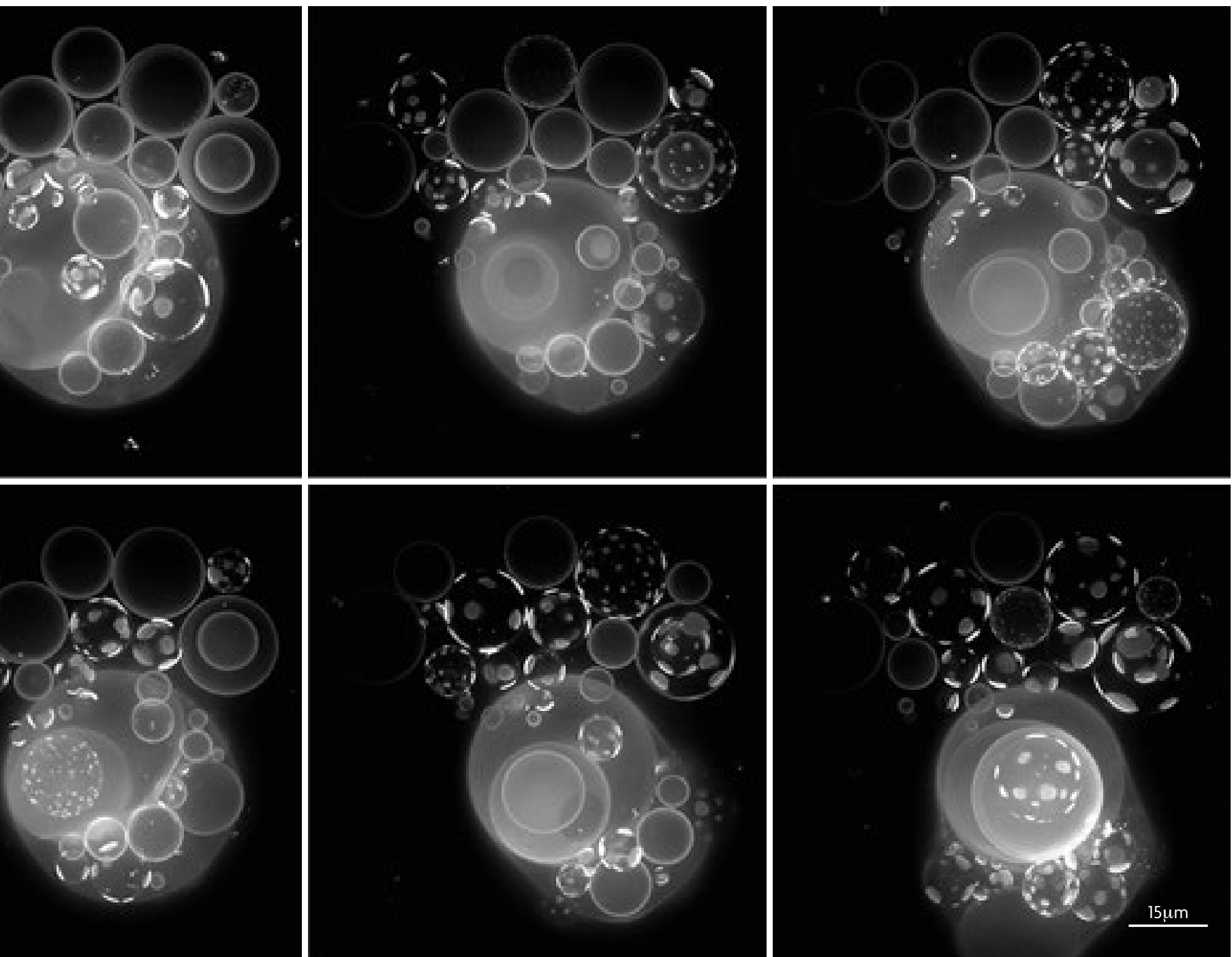

Figure 3. Time-resolved fluorescence microscopic images evidencing the complex dynamic behaviour that a population of liposomes can produce when responding to a simple externally-induced osmotic imbalance, through cyclic changes in the distribution of its membrane components.

«LIFE DOES NOT ONLY ORGANISE PRE-EXISTING MATERIAL, BUT ALSO CONTINUALLY GENERATES AND REGENERATES MOST OF IT AND, AT THE SAME TIME, CONTROLS MOST OF THE CONDITIONS IN WHICH SUCH A DYNAMIC ORGANISATION OCCURS» into account what science has contributed and the self-organisation paradigm, but also outgrows that information and gets closer to a more specific mode regarding the phenomenology of life (Keller, 2007).

From this perspective, one should focus, therefore, on researching different combinations of molecular components and interaction and transformation processes to find some that (while prebiotically plausible) obtained more cohesion, a more stable dynamic sustenance, as a possible condition for subsequent transitions. In fact, we consider it to be a very reasonable possibility that, in some environments on the Earth's surface 3,500 million years ago, there were many sets of chemical reactions distant from the equilibrium, which produced a great diversity of 
more or less stable compounds, driven by external energy sources (such as the Sun or geothermal energy, Figure 2). In this context, it is also reasonable to assume that part of these compounds and/or compound aggregates, when sufficiently accumulated (on an absorbent surface or in a micro-compartment), will have an effect on adjacent compounds and processes, so they can potentially contribute to the sustenance of the whole set of reactions (similar to a simple autocatalytic cycle in which every component of the cycle contributes to the synthesis of the rest). The idea is that those forms of cohesion and robust self-sustenance would be achieved when the influences between the different interacting components had the effect of reinforcing each other, avoiding natural tendencies like spatial dispersion or the decay of non-equilibrium material structures. It is not easy to determine the minimum set of components/ processes necessary to obtain an autonomous functional integration, so it is an issue that deserves to be investigated empirically. However, the presence of mechanisms for kinetic and spatial control (catalysts and compartments, respectively) and their correct coordination seem to be prerequisites.

In any case, what is interesting about these systems is that they would only be viable under certain external conditions (which could also make them disappear if the conditions were adverse enough). They would create and sustain most of the conditions that actually make their constitution and dynamic progression possible, because the presence of different components and their implication in the set of interaction and transformation processes makes the fulfilment of the rest possible. We are now at a stage where a radically new causal regime operates: molecule groups generate a set of material patterns and structures (other molecules or molecule aggregates, such as peptide chains with catalytic capacity or lipid compartments with selective permeability (see Figure 3), which constrain the underlying processes and transformations so they recursively regenerate those structures and, later, the set of interactions that dynamically sustain the entire system. This is an idea that other authors (Kauffman \& Clayton, 2006) have captured in terms of «constraint-work cycles», suggesting that constraint appears and spreads whenever there is a material configuration in a part of the universe that establishes these kinds of non-linear, recursive loops. They also stated that this is a key phenomenon in understanding the origin of life. It is, therefore, a matter of assembling a causal framework that, far from restricting or delimiting the space for possible dynamic states in the system, enables the existence of new stable unchanging states, reflecting the greater cohesion achieved between its components.

\section{IMPLICATIONS OF THE ALTERNATIVE APPROACH}

At this point, we are already in the position to answer Nagel's challenge. Matter managed to move from the physicochemical to the biological universe when it found, maybe also as a result of contingent events, stable ways of dynamic organisation arising from its ability for «self-constraint». These different organisations, based on a set of recursive, non-linear interaction loops, are not exclusive to biology: they appear spontaneously in the field of physical phenomena, as we can observe with hurricanes. But at some stage in the prebiotic process, already within a sufficiently rich interaction and transformation scenario

(that is, a necessarily chemical framework), the original feature was that some of the self-sustained systems could become self-productive, with a higher degree of internal cohesion and dynamic strength, when they generated multiple mutually-dependent constraints, which would, in turn, generate other ones, increasing in complexity and diversity. The basis for this increase in complexity lies in the constraints generated within the system, which open new organisational possibilities de facto when they selectively modulate the microscopic dynamics at the heart of the system.

In this context, self-sustaining can also start developing a new aspect: self-reproduction. In reality, self-reproduction is nothing more than a special form of self-production. But the emergence of life requires the self-sustaining systems to adopt that particular form of self-production that generates new units, because generating enough spatial proliferation 


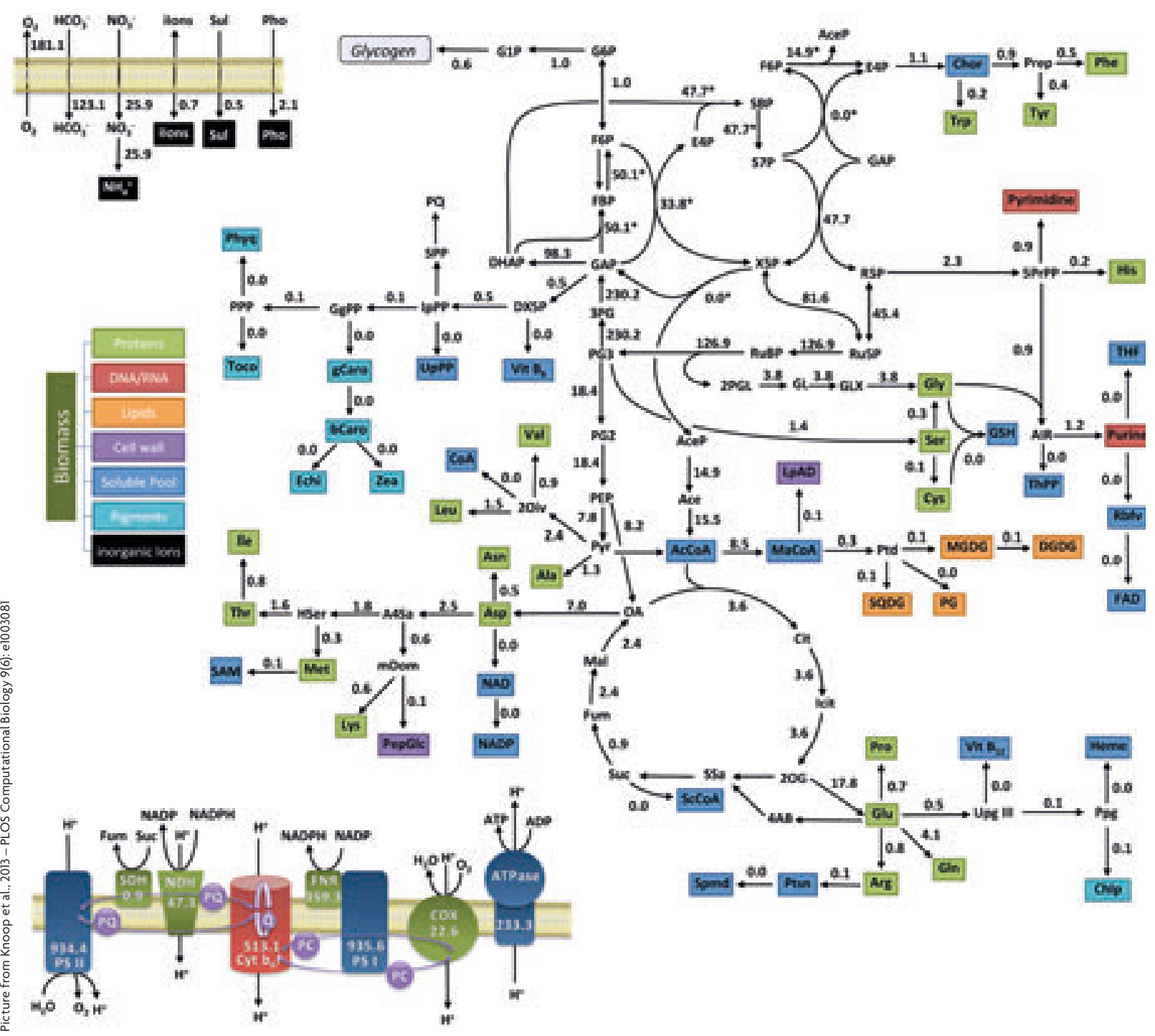

Figure 4. General simplified diagram on the quantity and variety of metabolic flows in an autotroph unicellular organism (in this case, a cyanobacterium in constant light radiation conditions). The diagram gives an idea of the enormous diversity of components in any living being, even the simplest ones, as well as the strong functional integration between the modules that constitute it.

and an indefinite temporal continuity (that is to say, an open history), based on the causal continuation between individual ephemeral organisations, is the only situation that can guarantee the fixation and eventual increase of random innovations. What fixes these innovations is simply the fact that they contribute to maintaining the individual organisations from which they stem. Therefore, their functionality and potential selective advantage can never be explained completely outside of the organisation framework. This, together with the fact that there is proliferation and hereditary transmission, is what fosters the beginning of a primitive form of evolution by natural selection.

In short, there is no «magic»-as claimed by Nagel - in the origin of life. There is no magic, but we are faced with an extraordinarily intricate phenomenon with profound scientific and 


\section{MONOGRAPH}

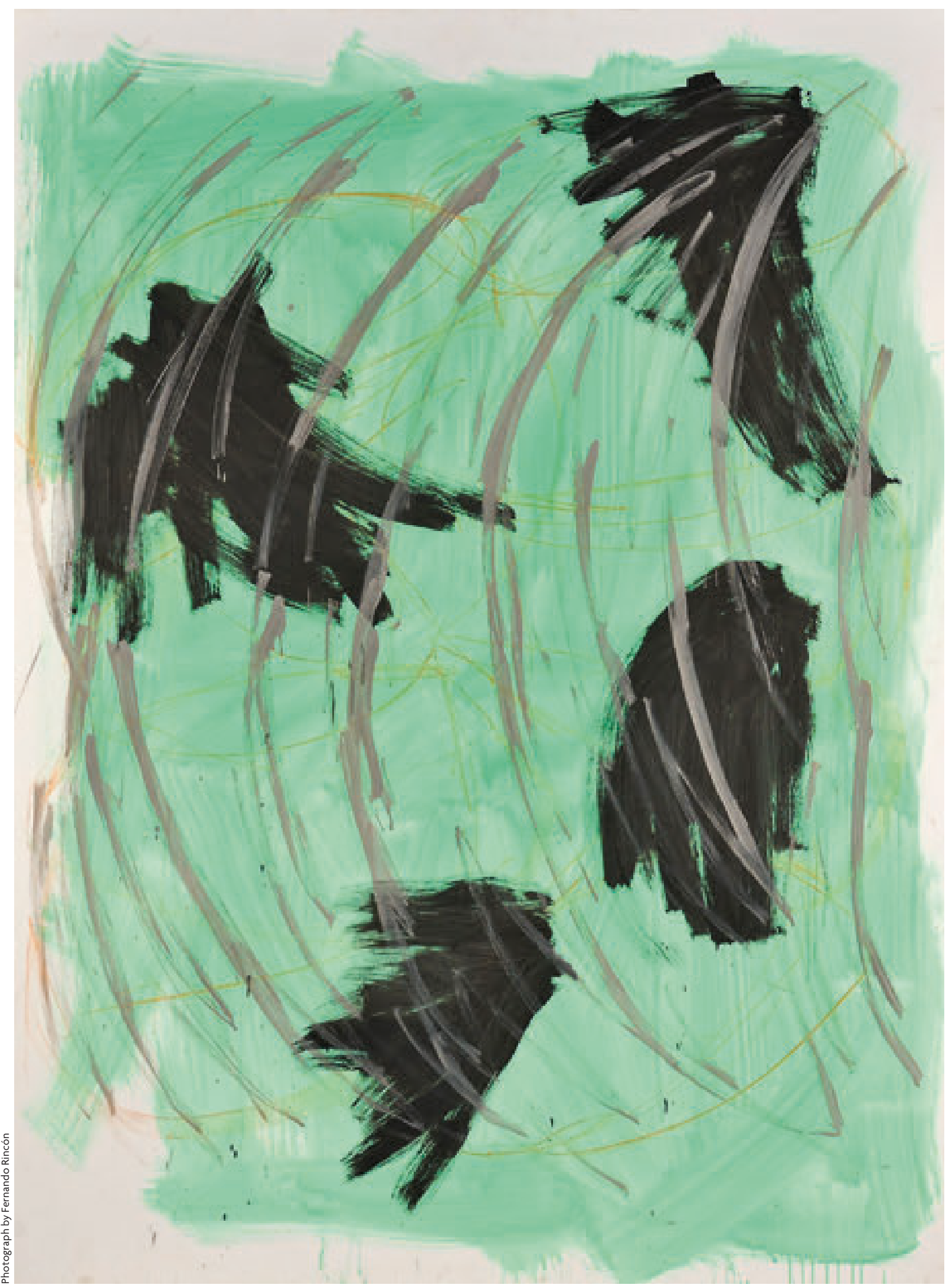

Rebeca Plana. Whiz-Whiz, 2014. Mixed technique on linen, $150 \times 200 \mathrm{~cm}$. 
philosophical implications, because it is inexplicable from the «usual» logic that physicists and chemists use to understand non-living phenomena. The emergence of strong self-sustaining material systems that can develop stable reproductive cycles (that is, genetically instructed metabolisms) brings along the establishment of complex chemical transformation loops (see Figure 4) that allow the integration of regulatory mechanisms (based on hierarchical control relationships between different functional modules or subsystems), together with reliable inheritance mechanisms (based on informational relationships). A general and consistent theory about biological organisation, if such a theory can be created, should explain the emergence of natural systems that generate and sustain these kinds of information and regulation architectures, taking into account the new results from system chemistry, biology, and synthetic biology. Here, we do not intend to act before we have the results, although we have tried to identify some of the fundamental concept issues we think will arise in the course of this enterprise, and the theoretical approach required to overcome them. Research on the origin of life, insofar as it tries to connect the fields of physics and chemistry with biology, and is forced to understand the urgency and interrelation of both aspects of the phenomenon (its individual organisation - that is, the emergence of cell metabolisms - and its collective evolutionary capacities - the historical process of diversification and the increase in complexity), constitutes a compulsory field for work on a future theory. In broader terms, of course, it is also to understand the relationships between different branches of established scientific knowledge. That is to say, until we solve the problem of the origin of life, we will not be able to accurately characterise the independence (or the forms of interdependence) of biology from physics and chemistry.

\section{ACKNOWLEDGEMENTS}

The authors would like to thank the two anonymous reviewers for reading the manuscript and suggesting improvements to the text. They would also like to thank the Basque government (IT 590-13), the Ministry of Economy and Competitiveness (FFI2011-25665 - KRM and AM) and the Ministry of Industry and Innovation (BFU2012-39816-C02-02 - AM) for their support to this research project. In addition, KRM is beneficiary of the European COST Actions CM1304 and TD1308.

\section{REFERENCES}

Eigen, M., \& Schuster, P. (1979). The Hypercycle: A principle of natural self-organization. New York: Springer.

Fry, I. (2000). The emergence of life on Earth: A historical and scientific overview. New Brunswick: Rutgers University Press.

Karsenti, E. (2008). Self-organization in cell biology. A brief history. Nature Reviews Molecular Cell Biology, 9, 255-262. doi: 10.1038/ $\operatorname{nrm} 2357$

Kauffman, S., \& Clayton, P. (2006). Agency, emergence and organization. Biology and Philosophy, 21, 501-521. doi: 10.1007/s10539-005-9003-9

Keller, E. F. (2007). The disappearance of function from 'selforganizing systems'. In F. Boogerd, J. H. Bruggeman, H. V. Hofmeyr, $\&$ H. Westerhoff (Eds.), Systems biology. Philosophical foundations. (pp. 303-318). Dordrecht: Elsevier.

Lewontin, R. (1970). The units of selection. Annual Reviews of Ecology and Systematics, 1, 1-18. doi: 10.1146/annurev. es.01.110170.000245

Monod, J. (1970). Le hasard et la nécessité. Essai sur la philosophie naturelle de la biologie moderne. Paris: Éditions du Seuil.

Moreno, A., \& Ruiz-Mirazo, K. (2009). The problem of the emergence of functional diversity in prebiotic evolution. Biology and Philosophy, 24(5), 585-605. doi: 10.1007/s10539-009-9178-6

Nagel, T. (2012). Mind and cosmos. Why the materialist neo-Darwinian conception of nature is almost certainly false. Oxford: Oxford University Press,

Orgel, L. E. (1992). Molecular replication. Nature, 358, 203-209. doi: $10.1038 / 358203 \mathrm{a} 0$

Pattee, H. H. (1969). How does a molecule become a message? Developmental Biology Supplement, 3, 1-16.

Peretó, J., Bada, J. L., \& Lazcano, A. (2009). Charles Darwin and the origin of life. Origins of Life and Evolution of Biospheres, 39(5), 395406. doi: 10.1007/s11084-009-9172-7

Ruiz-Mirazo, K., \& Moreno, A. (2012). Autonomy in evolution: From minimal to complex life. Synthese, 185(1): 21-52.

Simon, H. (1962). The architecture of complexity. Proceedings of the American Philosophical Society, 106, 467-482.

Szostak, J. W., (2012). The eightfold path to non-enzymatic RNA replication. Journal of Systems Chemistry, 3, 2. doi: 10.1186/1759-22083-2

Wicken, J. S., (1987). Evolution, thermodynamics and information. Extending the Darwinian program. Oxford: Oxford University Press.

Kepa Ruiz-Mirazo. Researcher at the University of the Basque Country (UPV/EHU). He focuses mainly on aspects of philosophical and scientific interest related to the origin and definition of life, at the cross-roads between physics, chemistry, and biology. He works in the Department of Science Logic and Philosophy (FICE), in Donostia-San Sebastián, and is also affiliated to the Science and Technology School in Leioia (Bizkaia, Spain). He is a specialist in proto-cellular models, and after receiving a Ramón y Cajal contract, he was awarded the I3 Certificate to recognise his research career, combining experimentation, computational simulations, and theoretical reflection.

Álvaro Moreno. Full professor of Science Philosophy at the University of the Basque Country (UPV/EHU). He created the Biology and Cognition Philosophy Research Group (IAS-Research Group). He is the author of more than 150 scientific publications (including two monographs and four edited volumes) and has made almost as many contributions to national and international conferences. He focuses on the philosophy of biology, artificial life, complex systems, and cognitive science, and his areas of interest encompass the philosophy of biology, general science philosophy, the study of complex systems, self-organisation, artificial life, and the origin of life and cognition. 